\title{
Anomalous Origin of Left Coronary Artery from Pulmonary Trunk in an Adult Patient -A Rare Occurrence
}

\author{
AW Chowdhury, MA Muttalib, MA Baqui, S Dutta, MM Alam, M Shakawatullah, DK Mondol, \\ S S Islam, MS Shahidullah, A HM Kamal \\ Department of Cardiology, National Institute of Cardiovascular Diseases, Dhaka
}

Key words: Anomalous origin of coronary artery, Pulmonary artery, Left coronary artery

\begin{abstract}
Anomalous origin of coronary arteries is a rare cause of cardiac disease. One of the very rare coronary anomalies is origin of left main coronary artery from pulmonary trunk. Most of these patients die in the first year of life and only 10-15\% survive in adulthood. We are reporting a rare case of anomalous origin of left coronary artery from pulmonary artery who survives into sixth decade of life.
\end{abstract}

(CVJ 2008; 1(1) : 117-121)

\section{Introduction}

Variation in the origin, course, or distribution of the epicardial coronary arteries are found in 1 to 2 percent of the population. Certain type of these anomaliesincluding ostial lesions, passage of major artery between the walls of the pulmonary trunk and aorta, or perhaps myocardial bridges-may produces ischemia with subsequent myocardial infarction. Considering anomalous origin of a coronary artery, in more than 90 percent cases the left main coronary artery is the anomalous artery; thus the anteroseptal and anterolateral left ventricular myocardium may be at jeopardy for injury. ${ }^{1}$

Most instances of this defect have been found in babies who have died in the first year of life. However, in some instances survival is possible into adult life. $^{2}$

Most common clinical presentations in the infants are angina like symptom, dyspnea, cyanosis, pallor, and occasional loss of consciousness ${ }^{3}$. Older children or adults usually present with a continuous murmur or with mitral regurgitation resulting from dysfunction of ischemic or infracted papillary muscles. In some instances the coronary anomaly is unsuspected until a previously well adolescent or adult experiences angina, heart failure, or sudden death.

Diagnosis can be made by electrocardiography, echocardiography, CT angiogram, stress thallium scintigraphy, positron emission tomography, and coronary angiography. Death may occur at any age up to 64 years or more, but the overwhelming majority do not survive the first year of life. Survivals appear to be related primarily to the amount of direct or indirect circulation reaching the left ventricle.

\section{Case Report}

A 60 years old man suffering from acute calculus cholecystitis was referred to the cardiologist by a

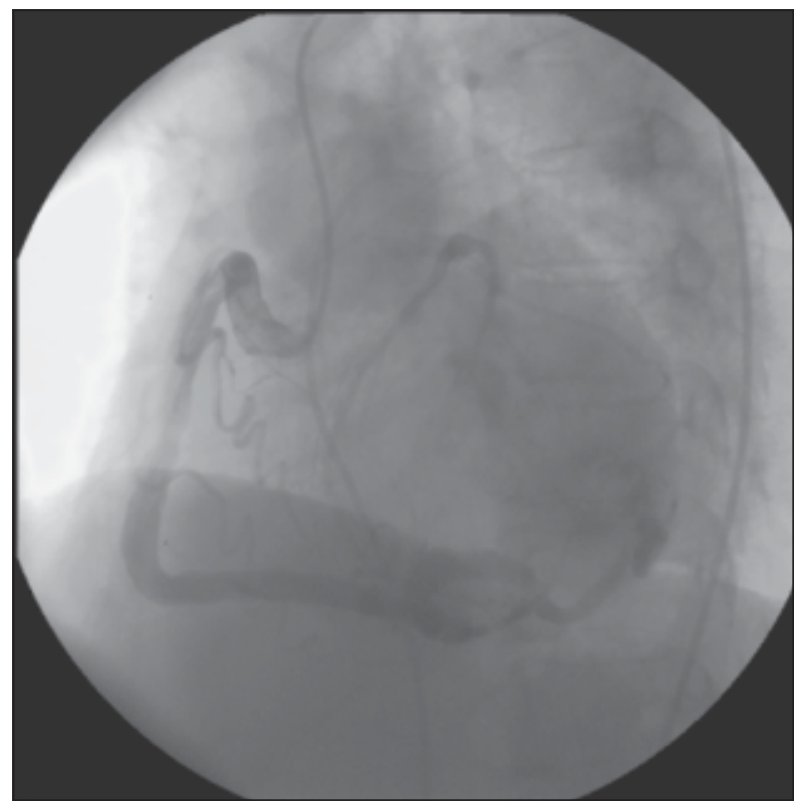

Fig.-1: Coronary angiogram showing right coronary artery drain by left coronary artery to pulmonary trunk

Address of Correspondence : Dr. A W Chowdhury, Associate Professor, Department of Cardiology, NICVD, Dhaka. 
surgeon. Surgeon sent this patient for cardiac evaluation because of gross ischemic changes in anterior leads in ECG and patient had effort intolerance (NYHA class-III). Patient was a known hypertensive for 7 years and was on tab. Methyl dopa, $250 \mathrm{mg}$ b.d. On echocardiographic evaluation, patient had dilated left ventricle with eccentric hypertrophy with anteroseptal hypokinesia and moderate left ventricular systolic dysfunction $(\mathrm{LVEF}=40 \%)$. Patient was very symptomatic due to acute calculus cholecystitis and needed urgent surgery but given his cardiac condition it was deemed to be a high risk case for any operative procedure. So, two weeks of intensive medical therapy was given for his cardiac problem and cholecystitis. Two weeks later, patient was reevaluated and he was found to be in very stable cardiac condition with NYHA class-I effort intolerance and his echocardiography also revealed remarkable improvement. There was only mild septal hypokinesia with EF improved to $48 \%$. Patient then underwent cholecystectomy with anesthesia given by a cardiac anesthetist. After 3 weeks he underwent elective coronary angiogram to evaluate his coronary status. During angiogram left coronary artery (LCA) could not be engaged and the origin of the left main artery could not be visualized. So, right coronary arteriogram was done first which showed a very dilated right coronary artery (RCA) with extensive collateralization to left system. The whole left system was visualized with blood draining from the left main coronary artery into the pulmonary artery. This was ascertained in different views which showed there was apparently no obstructive lesion in the left coronary system. On right coronary arteriogram delayed film could not clearly show the coronary sinus. Pulmonary arteriogram was done, which, however fail to visualize the LCA. The likely explanation for this is that pressure difference between the aorta and the pulmonary artery was very significant. The aorta pressure was 110/60/82 $\mathrm{mm} \mathrm{Hg}$ and the pulmonary artery pressure was 30/14/22 mm Hg. Keeping the NIH catheter in the pulmonary artery, right coronary angiogram clearly demonstrate that blood was draining from the right coronary artery through collaterals into the left coronary system and then into the pulmonary artery. Oxymetry showed right ventricular oxymetry was $61 \%$, in the pulmonary artery oxymetry was $66 \%$. This proved that there was excellent blood flow from the RCA to the LCA draining into the pulmonary artery. So, our diagnosis was anomalous origin of the LCA from the pulmonary artery. Post angiogram repeat echocardiography was done, it showed left ventricular hypertrophy, mild septal hypokinesia, a very large RCA (diameter-9 $\mathrm{mm}$ ), arising from aortic sinus and the LCA (diameter-4.9 $\mathrm{mm}$ ) arising from the pulmonary artery. For academic interest CT angiogram was also done (Fig. 2 \& 3). Which showed LCA originating from pulmonary artery. So, our final diagnosis is a very rare case of anomalous origin of the LCA from the pulmonary artery that has survived into adulthood.

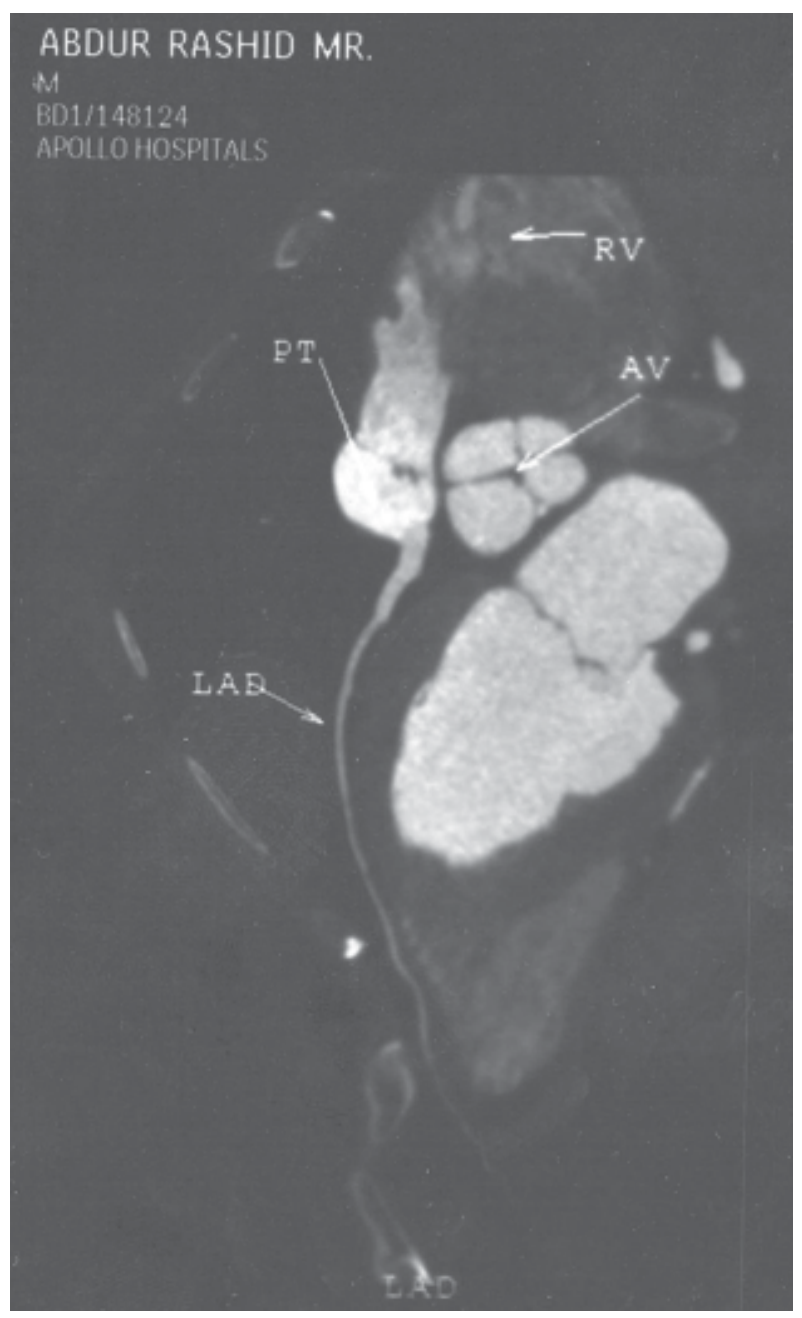

Fig.-2 : CT Angiogram showing origin of $L C A$ 


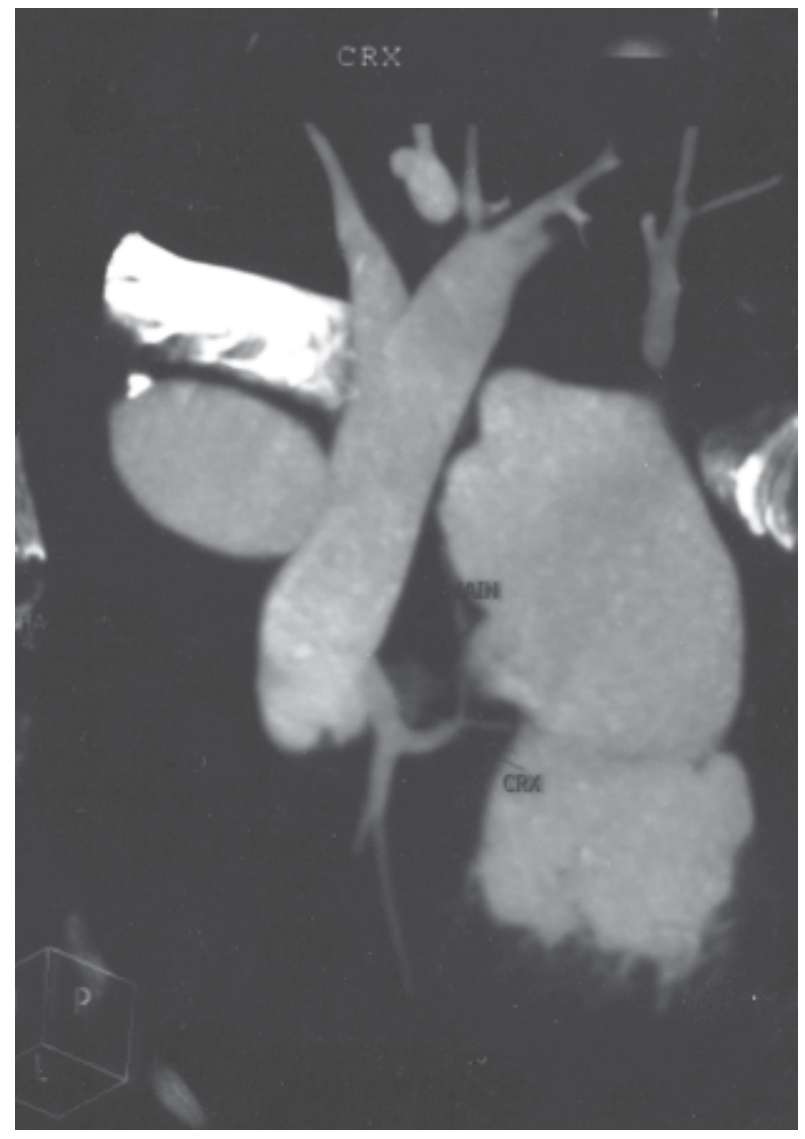

Fig.-3 : CT Angiogram showing origin of LCA

\section{Discussion}

The LCA arises from the superior portion of the left aortic sinus, just below the sinotubular ridge of the aorta. The left main ranges from 3 to $6 \mathrm{~mm}$ in diameter and may be up to 10 to $15 \mathrm{~mm}$ in length. The LCA courses behind the right ventricular outflow tract and usually bifurcates into left anterior descending artery and left circumflex artery. Rarely, the LCA is absent, and there are separate ostia of the left anterior descending artery and left circumflex artery. ${ }^{4}$

Anomalous pulmonary origin of the coronary artery occurs in about 0.24 percent of patients with congenital cardiac anomalies and occurs once in 300000 children. In almost all of the patients the LCA originates from the posterior sinus of the pulmonary artery. The anomalous origin of LCA from the pulmonary artery in a heart without other defects was originally described by Abriskoff in 1911. ${ }^{2}$ Since then sporadic cases has been reported intermittently.
Embryologically the distal coronary artery system is formed by 9 weeks from solid angioblastic buds that extend throughout the epicardium to form the major coronary artery branches. Proximally the coronary network form a ring around the truncus arteriosus, joining with coronary buds from the primitive aortic sinuses as the truncus partitions to form the great arteries. The varieties of anomalous pulmonary origin of the coronary artery are the result of displacement in this proximal process. $^{4}$

Before birth the oxygen content of the blood in the right and left ventricles is essentially the same. Furthermore, the pressures are similar. Among the cardiovascular adjustments that take place at, or shortly after, birth is a fall in pressure in the right ventricle and pulmonary artery, as compared to that in the left side of the heart. A coronary artery arising from the pulmonary artery will thus be receiving venous blood under low pressure; or the flow may be reversed, the collaterals from the RCA supplying the left ventricle and the LCA merely acting as a vein. If adequate collateral channels exists or develop between the two coronary artery circulations, total myocardial perfusion through the RCA increases ${ }^{5}$. In 10 to 15 percent of patients myocardial ischemia never develops because extensive intercoronary collaterals allow survival to adolescence or adulthood.

Infants with this anomaly appear normal at birth and, as a rule, continue to react as normal babies for the first month. They may even appear in good health until the second, third, or fourth month of life, but most frequently symptoms begin to appear in the second or third month of life. These symptoms may be grouped under three headings: (1) discomfort, (2) heart failure, and (3) respiratory infections. Feeding and defecation often are accompanied by irritability and crying, pallor, diaphoresis, and occasional loss of consciousness. The chief symptoms, however, are intimately associated with the onset of heart failure, and include dyspnea, tachycardia, wheezing respiration, cough, and occasionally, secondary cyanosis. The few cases that survive until adult life usually do not have signs or symptoms present during infancy or childhood. Older children or adults may present with features of ischemia, heart failure, and sudden death. 
The diagnosis of anomalous origin of the coronary artery is supported by the electrocardiographic appearances. In the majority of cases the electrocardiogram reveals a pattern that is associated with anterior myocardial infarction and almost invariably there is a $\mathrm{QR}$ pattern and inverted $\mathrm{T}$ wave in standard lead I and unipolar lead aVL. This is accompanied by deep $Q$ waves in $\mathrm{V}_{5}$ and $\mathrm{V}_{6}$. In 60 percent cases there are also inverted $\mathrm{T}$ waves in these leads. The majority of cases have evidence of a raised S-T segment over the left precordium followed by a $\mathrm{T}$ wave that is cove shaped and symmetric in outline. These findings greatly assist the distinction of this anomaly from myocarditis and dilated cardiomyopathy. ${ }^{6}$ Chest roentgenograms show moderate to severe enlargement of the left atrium and ventricle. Echocardiography may show left ventricular hypertrophy and wall motion abnormality. Doppler color flow mapping demonstrates retrograde flow in the left coronary systems and an abnormal flow jet from the LCA into the pulmonary artery. The origin of the anomalous LCA occasionally may be visualized from long and short axis views of the pulmonary artery. Doppler examination may also reveal associated mitral regurgitation. ${ }^{7} \mathrm{CT}$ angiogram shows anomalous origin of the LCA from the pulmonary artery. Stress thallium scintigraphy shows a characteristic defect of the anterolateral wall of the left ventricle. Positron emission tomography reveals both the perfusion defect and its metabolic consequences. Coronary angiography demonstrates the retrograde drainage of the coronary vessel into the pulmonary artery. The magnitude of shunting into the pulmonary artery may be determined by oxymetry, indicator dilution curves, or angiography.

Our patient had gross ischemic changes in anterior leads from $V_{1}$ and $V_{4}$. Echocardiography revealed left ventricular hypertrophy, mild septal hypokinesia, a very large RCA (diameter-9 $\mathrm{mm}$ ), arising from aortic sinus and the LCA (diameter$4.9 \mathrm{~mm}$ ) arising from the pulmonary artery, CT angiogram showed anomalous origin of the LCA from the pulmonary artery with normal origin of the RCA. The coronary sinus is normal in size and location. The pulmonary veins are normal. During coronary angiogram right coronary arteriogram showed a much dilated RCA with extensive collateralization to left system. The whole left system was visualized with blood draining from the left main coronary artery into the pulmonary artery.

The differential diagnosis includes those congenital heart defects that are associated with left ventricular hypertrophy, such as endocardial fibroelastosis, tricuspid atresia, aortic stenosis, patent ductus arteriosus, coarctation of the aorta, ventricular septal defect, myocarditis, or coronary calcinosis.

Medical treatment is indicated in infants with myocardial infarction for congestive heart failure, arrhythmia, and cardiogenic shock. Medical treatment with digitalis, diuretics, and oxygen should be initiated early and may postpone the fatal outcome for a short time while surgical management is being considered. The operations that have been used include reimplanting the LCA, surgically creating an aortopulmonary window and a tunnel to convey blood from the window across the back of the pulmonary artery to the anomalous LCA, with reconstruction of the anterior wall of the pulmonary trunk, and anastomosis of the LCA with the subclavian artery or with the aorta by means of a graft. ${ }^{8}$ Uncommonly, it is necessary to consider aneurysmectomy or mitral valve replacement. ${ }^{9}$

In our case, the patient survived into adulthood without much clinical symptom. However in the last few years, patient developed progressively increasing effort intolerance. With medical treatment he became almost symptom free with improvement of his left ventricu-lar function. So, in this case any surgical intervention deemed unnecessary.

\section{References}

1. Waller BF. Nonatherosclerotic coronary heart disease. in: Fuster V, Alexander RW, O'Rourke RA, Roberts R, King III SB, Nash IS, Prystowsky EC, editors. The heart. $11^{\text {th }}$ ed. New York; McGraw-Hill, Inc; 2004.

2. Keith JD. Diseases of coronary arteries and aorta. in: Keith JD, Rowe RD, Vlad P, editors. General cardiac 
disease. $3^{\text {rd }}$ ed. New York; Macmillan Publishing Co. Inc; 1978.

3. Celermajer DS, Sholler GF, Howman-Giles R, Celermajer JM. Myocardial infarction in childhood: Clinical analysis of 17 cases and medium term follow up of survivors. Br Heart J, 1991; 65: 332-6.

4. Friedman WF. Congenital heart disease in infancy and childhood. in: Braunwald E, editor. Heart disease. $5^{\text {th }}$ ed. New York; W B Saunders Company; 1997.

5. Hurwitz RA, Caldwell RL, Girod DA, Brown J, King H. Clinical and hemo-dynamic course of infants and children with anomalous left coronary artery. Am Heart J, 1989; 118: 1176-81.

6. Johnsrude CL, Perry JC, Cecchin F. Differentiating anomalous left coronary artery ori-ginating from the pulmonary artery in infants from myocarditis and dilated cardiomyopa-thy by electrocardiogram. Am J Cardiol, 1995; 75: 71-8.

7. Schmidt KG, Cooper MJ, Silverman NH, Stanger P. Pulmonary artery origin of the left coronary artery: Diagnosis by two-dimensional echocardiography, pulsed Doppler ultrasound and colour flow mapping. $J \mathrm{Am}$ Coll Cardiol, 1988; 11: 396-402.

8. Fernandes ED, Kadivar H, Hallman GL, Reul GJ, Otta DA, Cooley DA. Congenital malformations of the coronary arteries: Texas Heart Institute experience', Ann Thorac Surg, 1992; 54: 732-40.

9. Francois K, Jordaens L, Van Nooten GJ. Anomalous origin of the left coronary artery from the pulmonary artery. Ann Thorac Surg, 1993; 56: 1168-76. 